\title{
¿Es global la historia del arte de los Balcanes?*
}

IS BALKAN ART HISTORY GLOBAL?

É A HISTÓRIA DA ARTE DOS BALCÃS GLOBAL?

\section{Suzana Milevska** / Traducción Juan Carlos Arias***}

Cuadernos de Música, Artes Visuales y Artes Escénicas

/ Volumen 9 - Número 1 / Enero - Junio de 2014

/ ISSN 1794-6670/ Bogotá, D.C., Colombia / pp. 171-177

Fecha de recepción: 27 de febrero | Fecha de aceptación:

15 de septiembre de 2013. Encuentre este artículo en http://

cuadernosmusicayartes.javeriana.edu.co/

doi:10.11144/Javeriana.MAVAE9-1.ghab

* Publicado originalmente en Is Art History Global?, editado por James Elkins, Nueva York: Routledge, 2007, pp. 214-222.

* Profesora de la cátedra de Historias del Arte del Centro y Sur de Europa, Academia de Bellas Artes de Viena, Viena, Austria.

***Profesor, Departamento de Artes Visuales, Facultad de Artes, Pontificia Universidad Javeriana

Nota del editor: El presente texto proviene de la compilación de James Elkins denominada ¿Es la historia del arte global?, publicada por Routledge, Nueva York en 2007. Aquí la filósofa e historiadora del arte Susana Milevska da respuesta a una pregunta que será formulada a diversos investigadores historiadores y teóricos del arte en relación a si ven posible que se realice una historia del arte que sea global. 
En el título de este texto reformulo la pregunta que ha sido sugerida como punto de partida de esta discusión acerca de la situación actual de la historia del arte: “¿Es global la historia del arte?". Al hacerlo, me propongo señalar una serie de dificultades tanto en torno a esta pregunta tal como está formulada en el título del presente volumen, como en torno a sus absurdas implicaciones en diferentes contextos. Si uno pregunta "¿es global la historia del arte balcánico?", la pregunta misma se vuelve retórica, y es claro desde el principio qué respuesta sería necesaria.

Empecemos con la razón más obvia para la respuesta negativa: difícilmente existen historiadores del arte o libros de historia del arte de los Balcanes que sean conocidos en algún otro lugar diferente de su propia región. Un segundo punto estaría relacionado con el término "historia del arte balcánica": se sabe que los Balcanes no son una región homogénea. Debido a diversos conflictos militares y políticos en el pasado, los profesionales de los países que forman los Balcanes no se comunican fácilmente entre sí, y difícilmente tienen conocimiento de los textos de historia del arte producidos por los demás. De este modo, parte de esta segunda razón es que difícilmente se puede afirmar que exista una historia del arte balcánica como tal. La tercera razón, predecible tal vez, es que, obsesionados con sus propios problemas de definir qué es una historia del arte regional, nacional e internacional, los Balcanes no siempre siguieron el mismo ritmo de la historia del arte occidental. Sin embargo, es difícil generalizar pues puede ser una tarea compleja comparar las situaciones de la historia del arte en Grecia, Rumania, los países de la antigua Yugoslavia, Albania, y Turquía, al igual que comparar las historias del arte de distintos continentes. Los antecedentes históricos y políticos de todos estos países de alguna manera también han dado forma a sus historias intelectuales.

Es de esperarse que un absurdo similar emerja al intentar plantear la misma cuestión en otras partes del mundo. Las preguntas, ¿es global la historia del arte de Islandia?, ¿es global la historia del arte de Armenia?, ¿es global la historia del arte escandinava?, ¿es global la historia del arte americana?, plantean nuevas paradojas. Si la respuesta a cada una de estas preguntas es siempre negativa, ¿cómo se puede esperar que exista alguna historia del arte global que intente comparar, sintetizar y analizar todas estas historias del arte?

El único argumento racional aquí es que la pregunta busca, precisamente, la superación de cualquier límite o frontera entre estas diferentes historias del arte nacionales o regionales y, por lo tanto, pensar la posibilidad de una historia del arte para todas ellas. Algunas de estas historia no se prestarán a sí mismas para ser parte de una definición global simplemente porque ellas ya pertenecen a pequeñas entidades culturales cuyos miembros temen ser devorados por una única disciplina global. Algunas de ellas, como es el caso de la historia del arte balcánica (o, supongo, el caso de muchas otras regiones), ni siquiera están establecidas como tal dado que no existen espacios académicos e intelectuales que puedan formular los objetivos y alcances de tales estudios regionales. Los problemas de traducción y diferencias de idiomas, y los diversos sistemas de educación hacen que la pregunta inicial sea aún más compleja. Cuando me refiero al problema de la traducción no pienso necesariamente en la traducción al inglés. Sólo en los Balcanes existen cerca de veinte idiomas diferentes, y para tener una historia que abarque las artes de la región habría que suponer que todas ellas necesitan primero ser traducidas entre sí.

Con el fin de evitar la paradoja implícita en estas conclusiones, es necesario dar un paso atrás y preguntar qué es lo que haría global a la historia del arte. Tendrían que ser tomados en cuenta diversos criterios con el fin de definir de qué tipo de historia del arte global se debe 
hablar hoy. Si aceptamos que la trampa de la discusión entre una historia del arte occidental y una no-occidental no es muy productiva y se limita a ciertas preconcepciones de la historia del arte misma, una de las preguntas centrales sería: ¿existe una historia del arte que pueda ser global sin caer en dichas dicotomías?

Para enfrentar esta pregunta propongo tres temas que pueden ayudar tanto a aclarar el problema de pensar la historia del arte en términos globales, como a iluminar la posibilidad de encontrar una historia del arte que se ocupe del arte mundial. Discutiré las nociones de historicidad (el desarrollo histórico lineal del arte), traducción y algunos problemas relacionados con el sistema educativo.

\section{HISTORICIDAD}

Es difícil escribir y hablar acerca de la historia del arte hoy, en un momento en el que nadie parece creer que pueda existir una historia lineal. Hay dos tendencias distintas en el mundo académico de la historia del arte actualmente. Por un lado, los departamentos de historia del arte están desapareciendo rápidamente de los mapas curriculares de las universidades occidentales debido a la presión de establecer nuevos departamentos que se ocupen de algunos temas de la historia del arte, pero disfrazados en nuevas formas no-históricas. Los objetos de dichos estudios son temas y problemas en lugar de periodos históricos, estilos o historias del arte nacionales. Los estudios visuales, los estudios culturales, los estudios poscoloniales y la cultura visual, para mencionar unos pocos ejemplos, están creciendo en nuevos departamentos que se ocupan de problemas similares a los de la historia del arte, pero desde perspectivas transdisciplinarias más amplias. La historia del arte empieza a sonar como algo obsoleto o incluso innecesario.

Por otro lado, para proteger la historia del arte tal como ha sido concebida y estudiada desde sus inicios, como una disciplina autónoma, los departamentos de historia del arte que aún sobreviven tratan a menudo de conducirla hacia la dirección opuesta. Se han vuelto incluso más conservadores y se han adherido a las viejas maneras de enseñar historia del arte. Mantienen, por ejemplo, la división del arte en periodos históricos, estilos y movimientos con énfasis en los desarrollos nacionales y regionales. Estas tendencias son particularmente evidentes en las llamadas culturas e historias del arte "periféricas", por ejemplo en la historia del arte tal como es enseñada en los Balcanes.

La brecha entre los departamentos de historia del arte viejos o tradicionales (conservados por profesores que han estado allí desde el inicio de los departamentos, o por aquellos que representan una segunda generación) y los espacios recientemente establecidos (compuestos en su mayoría por jóvenes profesionales que han sido educados en el exterior) se hace cada vez más amplia.

La historicidad de la historia del arte es cuestionada a menudo como inapropiada, especialmente cuando se tiene en cuenta que las nuevas disciplinas y teorías (y yo añadiría los estudios de género, gay y lesbianas, y la teoría queer) se ocupan de la historia del arte desde su propia perspectiva. Una de sus principales metas es deconstruir la historia del arte como la conocemos y como la hemos estudiado, debido particularmente a su inhabilidad para abarcar las diferencias culturales, étnicas y de género.

Una razón para el reciente oscurecimiento de la historia del arte por parte de todas estas nuevas disciplinas y departamentos es que la historia del arte tradicional no permite que cier- 
tos asuntos culturales complejos hagan parte de su metodología. Ella se centra en el arte en términos aislados, es decir, como un fenómeno distinto de otras actividades culturales que le dan forma, como la cultural popular, los medios masivos y las políticas culturales. Del mismo modo, asume al arte como separado de problemas más complejos como los regímenes patriarcales de representación y el poder del Estado. Durante un largo tiempo la historia del arte se ha limitado a un registro de temas bastante reducido.

No es una sorpresa, entonces, que existan dificultades para conceptualizar la historia del arte en términos globales. Si la historia del arte aún depende de la periodización de estilos y movimientos, y si se concentra en fenómenos que han sido expresamente desarrollados con el fin de interpretar el arte occidental, es difícil imaginar que la historia del arte pueda operar en nuevos contextos con alguna precisión o relevancia.

Ofreceré un corto ejemplo que puede ilustrar los obstáculos que se pueden encontrar cuando se aplica la historia del arte occidental a otros contextos: la emergencia del arte moderno en diferentes partes de Europa, en particular en Macedonia. Hay diversas definiciones del término "modernismo" y distintos desacuerdos acerca de su momento de origen. No se trata de un término fijo, lo cual le garantiza cierta flexibilidad. Sin embargo, no encuentro que ninguna de sus definiciones sea aplicable al arte de Macedonia. Todo el periodo temprano en el que los estilos modernistas se diferenciaron de otros no existe. No se produjo ningún tipo de pintura secular de caballete en esta parte de Europa hasta la década de 1920. El arte se limitaba a la pintura al fresco, íconos, tallado en madera, tejidos y bordados. Sin embargo, a mediados del siglo XIX, los primeros fotógrafos empezaron a trabajar en la región. En distintas partes de los Balcanes esta brecha entre la primera pintura de caballete y las primeras fotografías es diferente, pero en el caso particular de Macedonia la pintura de caballete se inició mucho tiempo después de diversos estilos y periodos artísticos occidentales, y también tiempo después de que las primeras fotografías fueran tomadas. En este sentido, es posible comprender por qué la introducción del modernismo fue diferente allí a muchos otros lugares en los que la fotografía estaba disponible e influenciaba a la pintura.

Las firmas del primer fotógrafo conocido en Macedonia, Hadzi Koste, llamado "el pintor de frescos y fotógrafo", ha sido hallada en dos frescos en la iglesia de San Demetrio en Veles (1855) y en el monasterio de San Jorge en el pueblo de Cicevo (1860-1868). Éste es un ejemplo de cómo el medio de la pintura de fresco propio de la Edad Media era practicado en paralelo a la fotografía, y cómo el modernismo en los Balcanes eludió varios periodos de la historia del arte occidental' ${ }^{1}$. El asunto aquí es si la historia del arte como ha sido escrita puede ocuparse de este tipo de orden histórico específico sin calificarlo de retraso.

\section{TRADUCCIÓN}

El problema de la traducción en la historia del arte está, en cierto modo, relacionado con el problema de la historicidad y el retraso². Si la historia del arte occidental es entendida, como usualmente lo es, como un punto de referencia, no es sorpresivo que el asunto de la traducción produzca un efecto de retraso. Cuanto antes sea publicada la traducción de algún libro, más ampliamente ésta puede ser difundida en el mundo académico. Sin embargo, es importante subrayar aquí que la traducción de los libros de historia del arte de un idioma a otro es, más que cualquier otra cosa, un problema de traducción cultural. No se trata de un asunto 
de nombres, hechos o fechas, sino de diferentes conceptos del arte, contextos culturales y escenarios geográficos.

Académicos de países remotos que no son parte del mundo escolar occidental a menudo se quejan pues no tienen acceso a los últimos libros escritos en inglés y publicados por profesores de renombradas universidades en Occidente. Para mí, uno de los más grandes obstáculos para la globalización de la historia del arte es el enfoque en una sola dirección de traducción. Mi argumento es que la traducción cultural, a diferencia de la traducción lingüística, sólo es posible si funciona en direcciones recíprocas, y que la historia del arte no es una excepción a esta regla.

En la mesa redonda James Elkins menciona el conocido libro de Steven Mansbach Modern Art in Eastern Europe: From the Baltic to the Balkans, ca. 1890-1939 (1999). No se puede sino estar de acuerdo con el comentario de Elkins que afirma que las frecuentes comparaciones entre artistas orientales y occidentales hechas en el libro a menudo no aportan ninguna información contextual o relevante acerca de los artistas orientales. Uso este ejemplo sólo porque, desde mi perspectiva, esta limitación en el texto de Mansbach sobre el arte de Europa del Este se debe a que su única fuente y punto de partida habría sido la colección de libros de historia del arte occidental. Si hubieran existido libros de historia del arte oriental traducidos al inglés, él habría podido agregar más referencias locales y contextuales. Por supuesto que, incluso si esos libros hubieran sido traducidos, Mansbach probablemente se habría enfrentado a otro problema: el hecho de que continuamente, incluso en los libros escritos y publicados localmente, las comparaciones más frecuentes son (tal como ocurre en su libro) entre artistas orientales y occidentales.

(Vale la pena mencionar en este contexto que en los Balcanes y Europa del Este un gran número de traducciones de materiales relacionados con la historia del arte son realizados por los museos. Este tipo de materiales textuales y visuales son mucho más accesibles para Mansbach y otros investigadores extranjeros interesados en escenas locales. Sin embargo, no debe olvidarse que los museos a menudo tienen intereses y políticas distintos a los de los historiadores del arte, y que los investigadores extranjeros necesitan ser conscientes de las agendas particulares de los museos).

\section{SISTEMAS EDUCATIVOS}

Tanto el problema de la historicidad como el problema de la traducción están entrelazados en el marco de los sistemas educativos. ¿Cómo se puede esperar que la historia del arte sea una disciplina global sin que los sistemas educativos sean también globales? No entraré aquí en la discusión de los problemas éticos que implica el promover la globalización del sistema educativo, aún cuando este tema es bastante urgente. Los sistemas educativos son ampliamente discutidos en la teoría poscolonial y, aún cuando son relevantes para la discusión sobre la historia del arte, el tema requeriría más espacio del que supone el presente texto $^{3}$. Me limitaré a decir que la historia del arte no es y no puede ser global si se espera que la globalización acontezca únicamente a través de la escritura. La escritura es una parte orgánica del sistema educativo.

Quisiera terminar donde empecé, con los departamentos de historia del arte y su actitud hacia la historicidad, las historias del arte nacionales y la traducción. En 1980 fui testigo de 
un acontecimiento dramático en el Departamento de Historia del Arte en Skopje (en ese entonces parte de Yugoslavia, hoy Macedonia). Yo era estudiante de segundo año y fui invitada a una rueda de prensa dada por nuestros profesores (hoy la mayoría de ellos están retirados). La principal motivación para la rueda fue la publicación en un diario local de un ataque escrito por un ex estudiante de historia del arte del mismo departamento. Él había escrito una crítica severa argumentando básicamente que los profesores eran perezosos, y que no habían producido artículos o libros relevantes. Uno de los profesores, a los que se le había pedido manejar el delicado asunto y estuvo a cargo de la rueda de prensa, trajo a la sala un gran número de libros, revistas, resúmenes y otros materiales con el fin de probar que el artículo en el periódico era simplemente un ruin e ingenuo intento de venganza debido a algunos desacuerdos entre el estudiante y sus ex profesores. El número de documentos fue realmente asombroso y mi primera idea fue que el departamento era sorprendentemente productivo.

Lo que me impresionó en ese momento, y lo que sigue impresionándome ahora cuando pienso en este episodio, es que ni uno solo de los libros que se exhibieron dentro de ese montón era un intento por resumir el arte europeo del oriente y el occidente en un único volumen. Ni un solo libro o ensayo discutía algo diferente al arte de Macedonia, sin importar el periodo (antiguo, medieval o contemporáneo). Todos los textos exhibidos eran panoramas históricos generales centrados en temas nacionales como ciertos monumentos medievales, monografías sobre artistas contemporáneos o sobre arte macedonio en general, o libros de texto exclusivamente dedicados a artistas de Europa del Este. No había ni una sola traducción de un artículo o un libro escrito por un historiador del arte de renombre internacional (estoy de acuerdo, este tipo de traducciones deben ser hechas por traductores profesionales, pero los profesionales en historia del arte podrían tener por lo menos un sitio en las juntas editoriales, o ser invitados para escribir introducciones a estos textos). Es más, no había ni una sola traducción de algún libro escrito por alguno de mis profesores a otro idioma, ni siquiera a los idiomas vecinos -a alguno de los veinte idiomas balcánicos. Este episodio ocurrió hace veinticinco años. Muchas cosas han cambiado desde entonces, excepto esta última.

La historia del arte cambia. Ella se abre lentamente a otras disciplinas y a la temporalización no lineal. Ella utiliza diferentes metodologías que van más allá de la comparación entre arte occidental y no-occidental. El viejo chiste que dice que se puede reconocer que un conferencista tiene conocimientos de historia del arte si usa dos proyectores en sus charlas comienza a desaparecer, lo que implica que han empezado a ser introducidos distintos métodos educativos (claro, no en todas partes). Esta transformación interna al sistema educativo es necesaria para cualquier movimiento que tenga como meta la globalización.

Para mí, proyectos como Is Art History Global? son urgentes y necesarios, principalmente porque su mismo título muestra preparación y disponibilidad para preguntar, más que para ofrecer respuestas. Tal vez la razón por la que la historia del arte no es global hoy (creo que sería ingenuo pensar que lo es) es el resultado de cierta tendencia de la historia del arte anterior que tenía todas las respuestas siempre a mano, incluso antes de ser escrita, y estaba basada en la limitada experiencia de sus escritores.

Para concluir, la historia del arte global puede ser provechosa sólo si traduce e incorpora todo conocimiento de historia del arte local que sea accesible. Tal vez una de las metas más importantes de la historia del arte global es transformar las historias del arte locales en partes de una herencia "glocal" 4 . Si las preguntas sobre la historicidad y la traducción 
se hacen más claras a la luz de los estudios culturales y poscoloniales, y si esos estudios han ayudado a la historia del arte a aclarar sus propios objetivos, el sistema educativo debe continuar creando modelos de enseñanza nuevos y más flexibles que puedan elevar la historia del arte a un nivel global.

\section{NOTAS}

1 Fotografías de ambas firmas fueron reproducidas en la revista Kinopsis [Skopje] 16 (1996): 122.

2 Maria Todorova definió a los Balcanes como determinados por los problemas de retraso, "sentido de atraso y falta" (Todorova, 2005, p. 145). En su artículo, Todorova se centra en el problema del retraso como tropo dominante en la historiografía de Europa del Este, especialmente en el discurso nacionalista hasta el final del siglo XX

3 Aquí me refiero a algunas observaciones críticas recientes sobre la globalización como un fenómeno cultural y político problemático desde una perspectiva ética, como por ejemplo la de Arjun Appadurai: “La globalización de la cultura no es lo mismo que su homogeneización. Pero la globalización implica el uso de una variedad de instrumentos de homogeneización que son absorbidos por las economías culturales y políticas locales" (2000, p. 42).

4 Para una corta pero provocativa discusión sobre la popularidad del concepto "glocal", ver el texto de Bruno Latour "On the Difficulty of Being Glocal".

\section{REFERENCIAS}

Appadurai, Arjun. Modernity at Large: Cultural Dimensions of Globalization. Minneapolis: University of Minnesota Press, 2000.

Kinopsis. Skopje, núm. 16 (1996): 122.

Latour, Bruno. "On the Difficulty of Being Glocal." Domus (marzo de 2004). www.ensmp.fr/ latour/ presse/presse_art/GB-02 Domus 02-04.html (Acceso: febrero de 2006).

Mansbach, Steven. Modern Art in Eastern Europe: From the Baltic to eh Balkans, ca. 1890-1939. Cambridge MA: University Press, 1999.

Todorova, Maria. "The Trap of Backwardness: Modernity, Temporality, and the Study of Eastern European Nationalism". Slavic Review, vol. 64, no. 1 (Primavera 2005): 140-64.

\section{Cómo citar este artículo:}

Arias, Juan Carlos. Traducción de “ ¿Es global la historia del arte de los Balcanes?" de Milevska, Suzana. Cuadernos de Música, Artes Visuales y Artes Escénicas, 9 (1), 171-177, 2014. http:// dx.doi.org/10.11144/Javeriana.MAVAE9-1.ghab 
PRESENTACIÓN DE OBRA 